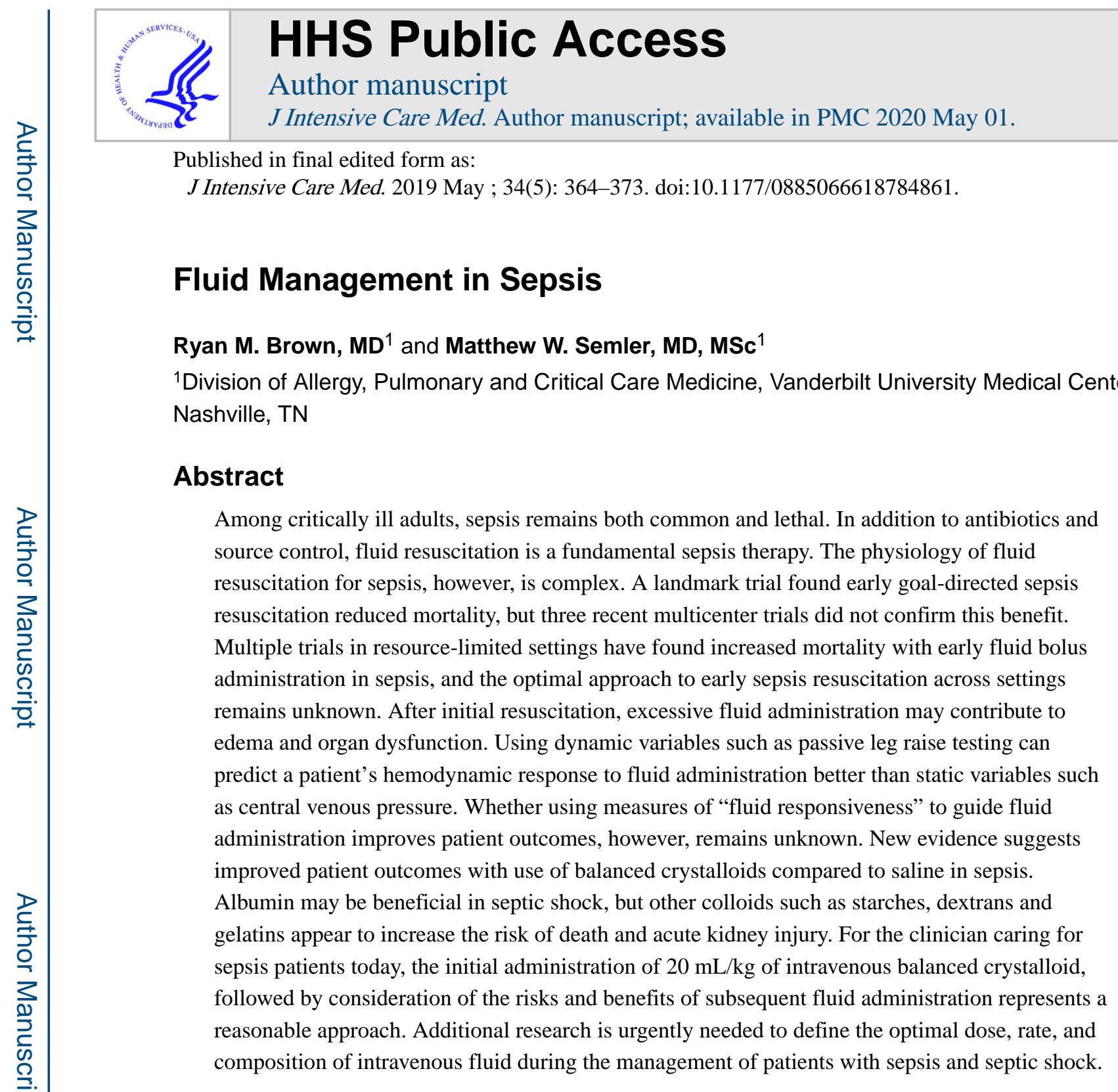

\title{
INTRODUCTION
}

Sepsis, a dysregulated host response to severe infection, accounts for 2-6\% of all hospital admissions and carries an in-hospital mortality of up to $15 \% \cdot{ }^{1-3}$ Mortality is even greater when sepsis is accompanied by hypotension and hypoperfusion (septic shock). ${ }^{3}$ Guidelines for sepsis management recommend early administration of antibiotics and intravenous (IV) fluid in addition to source control. Despite multiple recent clinical trials examining fluid management in sepsis, fundamental questions about which intravenous fluid to administer and in what amount remain unanswered. This article summarizes the physiologic principles and scientific evidence currently available to help clinicians make decisions regarding fluid management for patients with sepsis.

Corresponding Author: Matthew W. Semler, MD, MSc, $116121^{\text {st }}$ Ave S., C-1216 MCN, Nashville, TN 37232-2650, matthew.w.semler@vanderbilt.edu.

Authors contributions: Drafting of the manuscript: R.M.B.; Critical revision of the manuscript for important intellectual content:

R.M.B., M.W.S. 


\section{PHYSIOLOGY OF FLUID ADMINISTRATION}

Patients with sepsis experience altered oxygen delivery and extraction, in part due to varying degrees of actual and relative intravascular volume depletion from decreased oral intake, increased insensible losses, sepsis-induced vasodilation, increased venous capacitance, and capillary leakage. The classic understanding is that during early sepsis most patients experience "relative hypovolemia" and the administration of intravenous fluid increases preload, which increases cardiac output, resulting in improved oxygen delivery to organs experiencing tissue hypoxia (Figure 1). This classic understanding is increasingly recognized to be overly simplistic. There are many factors that influence tissue oxygen delivery and extraction other than hemodynamics. In addition, the hemodynamic response to intravenous fluid is determined by an intricate interaction of mean systemic filling pressure, right atrial pressure, venous resistance, ventricular compliance, and afterload. ${ }^{4}$ Fluid administration may affect many of these components, some of them deleteriously (e.g., fluid administration may decrease venous return by increasing right atrial pressure). ${ }^{5,6}$ The complexity of patients' responses to fluid administration in sepsis is evidenced by numerous studies reporting that approximately half of patients with sepsis do not experience hemodynamic improvement after fluid bolus administration, and that right atrial pressure poorly predicts hemodynamic improvement with intravenous fluid administration. ${ }^{7-9}$ Moreover, the century-old Starling model conceptualizing maintenance of vascular volume as the balance of hydrostatic and oncotic pressure gradients between the vessel lumen and interstitial space has been challenged by the recent recognition of the importance of the endothelial glycocalyx. ${ }^{10}$ Because it is a primary determinant of membrane permeability, damage to the glycocalyx during sepsis may alter patients' response to fluid resuscitation. Although the clinical implications of these findings are not yet fully understood, they argue against an overly simplified approach to understanding the effects of fluid composition and dose in sepsis.

\section{INITIAL SEPSIS RESUSCITATION}

Fluid administration is considered a fundamental part of early sepsis treatment. ${ }^{1}$ In the landmark Early Goal-Directed Therapy (EGDT) trial, ${ }^{11}$ Rivers and colleagues compared usual care to a protocolized approach to sepsis resuscitation using intravenous fluids, vasopressors, and blood transfusion among 263 patients in a single emergency department. In the usual care group, patients received arterial and central venous catheterization and were administered IV fluid to maintain a central venous pressure (CVP) of 8-12 $\mathrm{mm} \mathrm{Hg}$, and vasopressors to maintain mean arterial pressure (MAP) $\searrow 65 \mathrm{~mm} \mathrm{Hg}$. The EGDT group used the same hemodynamic targets, but additionally received continuous monitoring of central venous oxygen saturation, with blood transfusion for a hematocrit less than $30 \%$ and dobutamine administration to achieve a central venous saturation $\geq 70 \%$. During the 6 hours of intervention, EGDT patients received more IV fluid (mean 5.0 vs $3.5 \mathrm{~L} ; \mathrm{P}<0.001$ ), blood transfusions $(64.1 \%$ vs $18.5 \%$; $\mathrm{P}<0.001)$, and dobutamine $(13.7 \%$ vs $0.8 \%$; $\mathrm{P}<0.001)$. Inhospital mortality was $16 \%$ lower with EGDT compared to usual care $(46.5 \%$ vs $30.5 \% ; \mathrm{P}=$ 0.009). 
The remarkable improvement in mortality reported in this landmark trial led to incorporation of goal-directed fluid resuscitation into the recommendations for early sepsis management in the Surviving Sepsis Campaign (SSC) Guidelines. ${ }^{12}$ For more than a decade, this approach to sepsis fluid management was recommended in international guidelines, consensus statements, and hospital quality metrics. Three recent large, multicenter trials, however, did not report a benefit to EGDT compared to current usual sepsis care. The ProMISE ${ }^{13}$ $(n=1243), \operatorname{ARISE}^{14}(n=1588)$, and ProCESS ${ }^{15}(n=1341)$ trials all compared usual care to EGDT protocols based on the original EGDT trial. All three failed to demonstrate a benefit with EGDT (or protocolized usual care in ProCESS). A patient-level meta-analysis of these three trials ${ }^{3}$ also found no mortality benefit to EGDT. All-cause mortality at 90 days was $24.9 \%$ with EGDT and $25.4 \%$ with usual care $(\mathrm{P}=0.97)$. Furthermore, the meta-analysis reported that EGDT increased the length of stay in the intensive care unit (5.3 vs 4.9 days; $\mathrm{P}$ $=0.04$ ), the duration of cardiovascular support (1.9 vs 2.9 days; $\mathrm{P}=0.01$ ), and the cost of the hospitalization.

Several factors may account for the difference in results between the Rivers trial and the three recent trials of EGDT. First, the Rivers trial was a small, single center trial and therefore prone to type I error. Second, the difference between the EGDT and usual care groups in volume of fluid administered was modest in all three recent trials. In ProCESS, the trial with the largest separation between groups, there was a $1.1 \mathrm{~L}$ difference in fluid administered in the first 6 hours after enrollment, which falls short of the $1.5 \mathrm{~L}$ difference seen in the original Rivers study (Figure 2). Third, patients in the recent three trials were enrolled later after presentation to the Emergency Department, potentially missing a key period of early intervention. Fourth, the significantly lower mortality rates in the modern trials could imply that they enrolled less severely ill patients than the original trial, limiting the potential benefit of EGDT. Ultimately, the many changes in critical care and sepsis management over the decade and a half between the original EGDT and the three recent trials may preclude direct comparison of the studies and a conclusive understanding of the implications for optimal fluid management in early sepsis.

A common feature of these landmark sepsis fluid management trials is that they were all conducted in the high-income countries. In comparison, the Fluid Expansion as Supportive Therapy (FEAST) study randomized African children with sepsis-related hypoperfusion to a bolus of 5\% albumin, a bolus of saline, or no intravenous fluid bolus. ${ }^{16}$ Mortality in both the albumin and saline groups was significantly higher than in the group not treated with a fluid bolus (10.6\% vs $10.5 \%$ vs $7.3 \%$; P = 0.003). Similarly, the Simplified Severe Sepsis Protocol (SSSP) and Simplified Severe Sepsis Protocol-2 (SSSP-2) trials examined early fluid management for adults with sepsis in a limited-resource setting, without the routine availability of intensive care unit (ICU) beds or mechanical ventilation. The SSSP trial randomized adult patients in Zambia with severe sepsis to usual care or to 6 hours of protocolized care, which consisted of early fluid boluses, vasopressors to maintain a target MAP, and whole blood transfusions to maintain a target hemoglobin. ${ }^{17}$ The trial was stopped early by the data and safety monitoring board out of concern that patients with hypoxemic respiratory distress at baseline experienced higher mortality with protocolized care $(100 \%$ vs $70 \% ; \mathrm{P}=0.09$ ). The SSSP-2 trial attempted to mitigate this risk by excluding patients at high risk for respiratory failure and stopping fluids if there were signs of worsening 
respiratory function. ${ }^{18}$ Patients receiving protocolized care again received larger volumes of intravenous fluid in the first 6 hours (median 3.5 vs $2.0 \mathrm{~L} ; \mathrm{P}<0.001$ ) and experienced a larger decrease in serum lactate concentration $(-1.2 \mathrm{vs}-0.5 \mathrm{mmol} / \mathrm{L} ; \mathrm{P}=0.02)$. However, despite excluding patients with baseline hypoxemia and tachypnea, patients treated with the sepsis protocol experienced a higher rate of respiratory decline (35.8\% vs $22.3 \% ; \mathrm{P}=0.03$ ) and in-hospital mortality ( $48.1 \%$ vs $33.0 \%$; RR $1.46 ; \mathrm{P}=0.03)$. The detrimental effects of fluid bolus administration on clinical outcomes in these three trials may derive from lack of access to mechanical ventilation, patient factors such as severe malnutrition and hypoalbuminemia, or organism-specific factors from infection with malaria or tuberculosis. Alternatively, the effects of fluid administration in early sepsis on clinical outcomes may be similar in high- and low-resource settings, but the effects have only been observed in lowresource settings in which current usual care does not involve early fluid bolus administration. Together, the FEAST, SSSP, and SSSP-2 trials mandate caution with fluid bolus administration for patients with severe infection in resource-limited settings and intensify the urgency for understanding the relationship between early fluid administration and clinical outcomes of sepsis for patients across settings.

Current Surviving Sepsis Guidelines recommend early administration of $30 \mathrm{~mL} / \mathrm{kg}$ of intravenous fluid for septic shock or sepsis-induced hypoperfusion, which may manifest as "acute organ dysfunction and/or \pm decreased blood pressure and increased serum lactate."19 Patients in modern trials of EGDT and usual care received a median of $27 \mathrm{~mL} / \mathrm{kg}$ of fluid prior to randomization, slightly less than that recommended by the current SSC Guidelines. ${ }^{3}$ Given the potential harms of fluid resuscitation-induced volume overload, and the currently available evidence linking early fluid resuscitation to negative outcomes in resource-limited settings, we recommend a slightly more conservative initial bolus of $20 \mathrm{~mL} / \mathrm{kg}$ of intravenous fluid for patients with sepsis-induced hypotension or septic shock. Careful monitoring of patients' respiratory and hemodynamic function is necessary, as one-third to one-half of patients will experience persistent hypotension after initial fluid bolus administration, requiring clinicians to weigh the risks and benefits of further fluid administration or initiation of vasopressors. A currently-enrolling, large, multicenter randomized trial (CLOVERS, NCT03434028) comparing liberal to restrictive fluid management in early sepsis will provide valuable information on this fundamental question in sepsis management.

\section{FLUID ADMINISTRATION AFTER INITIAL RESUSCITATION}

After the initial resuscitation period, some patients may benefit from ongoing fluid administration to optimize hemodynamics and augment organ perfusion. However, in the days following initial sepsis resuscitation, many patients develop a net positive fluid balance, which can have negative consequences due to increased intracardiac pressures, organ edema, arterial vasodilation, and damage to the endothelial glycocalyx. ${ }^{6}$ Patients in the EGDT group of the original Rivers trial received an average of $13 \mathrm{~L}$ fluids in the first 72 hours. ${ }^{11} \mathrm{~A}$ retrospective analysis of the VAsopressin in Septic Shock Trial (VASST), which included 778 patients with septic shock on norepinephrine, showed that patients were, on average, 11 L net positive after 4 days of treatment. ${ }^{20}$ After correcting for age and APACHEII score, increasing net fluid balance at both 12 hours and 4 days correlated with increased mortality. 
Similarly, in a retrospective study of 2,632 ICU patients with severe sepsis and septic shock, each $1 \mathrm{~L}$ increase in cumulative fluid balance at 72 hours was independently associated with increased hospital mortality (aOR 1.06; P <0.001). ${ }^{21}$

These findings raise the question of whether patients with sepsis may benefit from a more restrictive fluid strategy after initial resuscitation. The Fluid And Catheter Treatment Trial (FACTT) compared conservative to liberal fluid management among 1,000 mechanically ventilated patients with acute respiratory distress syndrome, $70 \%$ of whom had sepsis or pneumonia. In the conservative fluid management group, patients without shock in the prior 12 hours were given furosemide to achieve a goal CVP $<4 \mathrm{~mm} \mathrm{Hg}$. The mean cumulative fluid balance during the first 7 days was $-136 \mathrm{~mL}$ in the conservative group and $6,992 \mathrm{~mL}$ in the liberal group $(\mathrm{P}<0.001)$. Patients in the conservative group experienced more ventilatorfree days (14.6 vs 12.1 days; $\mathrm{P}<0.001$ ) and ICU-free days (13.4 vs 11.2 days; $\mathrm{P}<0.001$ ) during the first 28 days. There was no increase in shock or receipt of renal replacement therapy with the conservative fluid strategy. A secondary analysis of FACTT found that, among patients with a low initial CVP ( $\$ 8 \mathrm{~mm} \mathrm{Hg}$ ), use of conservative fluid management also appeared to improve in-hospital mortality compared with liberal fluid management $(17 \% \text { vs } 36 \% ; \mathrm{P}=0.005)^{22}$.

No large trials have specifically compared liberal versus conservative fluid management after initial resuscitation among patients with sepsis or septic shock. The recently completed Conservative vs Liberal Approach to fluid therapy of Septic Shock in Intensive Care (CLASSIC) trial randomized 151 patients with septic shock after initial resuscitation to a conservative resuscitation strategy consisting of $250-500 \mathrm{~mL}$ crystalloid boluses for signs of severe hypoperfusion compared to a liberal strategy of crystalloid boluses permitted as long as hemodynamic variables continued to improve with fluid. ${ }^{23}$ Resuscitation fluid volume by day 5 was lower in the restrictive group (mean difference $-1.2 \mathrm{~L} ; \mathrm{P}<0.001$ ), but total fluid received was similar between groups. Mortality was similar between groups, however the conservative fluid group experienced less acute kidney injury (AKI). Plasma lactate levels, doses of norepinephrine, and urine output did not differ between groups. ${ }^{24}$

The above evidence highlights the uncertainty surrounding the optimal approach to fluid administration during and following sepsis resuscitation. In the absence of evidence from large randomized trials, many clinicians have gravitated towards attempting to guide fluid therapy by a patient's expected hemodynamic response to fluid therapy. Conceptually, distinguishing a priori patients likely to experience hemodynamic improvement with fluid administration ("fluid responsive") from those unlikely to experience hemodynamic improvement (not "fluid responsive") might help clinicians avoid the negative effects of fluid therapy for those patients who would not experience a hemodynamic benefit. The clinical appeal of this concept has led to a recent outpouring of research on measures of "fluid responsiveness." Static parameters once thought to predict patients' hemodynamic response to fluid administration (e.g., central venous pressure, ${ }^{8}$ mixed venous oxygen saturation ${ }^{25}$ ) have been increasingly supplanted by so-called dynamic variables. Dynamic variables are the changes in measurable hemodynamics (cardiac output, stroke volume, etc.) accompanying mechanical ventilation, small fluid challenges, or passive leg raise, and they more accurately identify patients who will experience increases in cardiac output or stroke 
volume in response to fluid administration. ${ }^{7,26}$ Dynamic variables that have been reported to identify "fluid responsiveness" include pulse pressure variation (PPV), ${ }^{26-28}$ stroke volume variation (SVV), ${ }^{26,29}$ systolic pressure variation (SPV), ${ }^{26}$ plethysmography variability index, 29 change in peak doppler arterial velocity, ${ }^{29}$ inferior vena cava (IVC) collapsibility/ distensibility, ${ }^{29,30}$ and cardiac output monitoring. ${ }^{31}$ Though these variables may outperform static predictors, in many cases their use is limited by the presence of spontaneous respiration or arrhythmias. Passive leg raise testing, however, can be used in most critically ill adults, even those with spontaneous breathing or cardiac arrhythmias. Raising a patient's legs to 45 degrees above horizontal causes $250-300 \mathrm{~mL}$ of blood to return from the venous system of the lower extremities to the heart. According to a recent meta-analysis, when this increased blood return results in an increase in stroke volume of at least $10-15 \%$, patients are likely to increase their cardiac output if they are subsequently administered an intravenous fluid bolus. ${ }^{7}$

Although measures of fluid responsiveness are increasingly able to identify patients who will experience increased cardiac output or stroke volume with the administration of an intravenous fluid bolus, whether administering intravenous fluid to patients who are "fluid responsive" improves outcomes remains completely unknown. ${ }^{32,33}$ The current Surviving Sepsis Campaign Guidelines recommend continuous monitoring of hemodynamics and use of dynamic variables predictive of "fluid responsiveness" to guide fluid resuscitation. Whether outcomes of sepsis are best with a liberal fluid strategy, a conservative fluid strategy emphasizing early vasopressors, or an approach in which fluid administration is guided by "fluid responsiveness" requires evaluation in high-quality randomized trials.

\section{CHOICE OF INTRAVENOUS FLUID}

The decision to administer IV fluids in sepsis logically leads to the question of "which fluid?" The ideal fluid for sepsis would expand intravascular volume without leading to tissue edema, increase cardiac output, contain a chemical composition similar to plasma, and improve patient-centered outcomes in a cost-effective manner. Unfortunately, this fluid does not currently exist. What follows is a discussion of the various fluids that are available for intravenous administration, their compositions (Table 1), and the current evidence regarding their respective effects on patient outcomes.

\section{Crystalloids}

Crystalloids are solutions of ions that are able to pass through semipermeable membranes such as capillaries. Crystalloids are the most commonly administered intravenous fluids in ICUs overall, and the most commonly administered crystalloid is saline $(0.9 \%$ sodium chloride). ${ }^{34-36}$ Isotonic crystalloids (e.g., saline, lactated Ringer's, Plasmalyte) have a tonicity similar to plasma. Both hypotonic (5\% dextrose in water, $0.45 \%$ sodium chloride) and hypertonic solutions (3\% sodium chloride) exist, but are not routinely used for fluid therapy in sepsis. Additionally, a recent trial of hypertonic saline in adults with sepsis did not demonstrate improved outcomes. ${ }^{37}$

Isotonic crystalloids may be categorized as "balanced" or "unbalanced." Saline is the prototypical "unbalanced" crystalloid, with a chloride concentration of $154 \mathrm{mmol} / \mathrm{L}$ (around 
$50 \%$ greater than the chloride content of extracellular fluid) and no organic anion to act as an acid-base buffer (Table 1). In contrast, balanced crystalloids such as lactated Ringer's, Hartmann's solution, Plasmalyte, or Normosol contain a chloride concentration more similar to that of plasma (98-112 mmol/L). To achieve this, most balanced crystalloids substitute an organic anion such as bicarbonate, lactate, acetate, or gluconate in place of chloride resulting in a more neutral $\mathrm{pH}$ and a greater strong ion difference.

Decades of pre-clinical research and studies in the operating room have shown that use of fluids containing high chloride concentrations may lead to hyperchloremic metabolic acidosis, ${ }^{38-43}$ decreased renal perfusion, ${ }^{41}$ acute kidney injury, ${ }^{44}$ altered inflammatory cascade, ${ }^{45}$ and reduction in arterial blood pressure ${ }^{39}$ compared to use of balanced crystalloids. Observational studies among critically ill adults suggested that use of balanced crystalloids might reduce the risk of death. ${ }^{46-48}$

These preliminary data provided the rational for four recent randomized trials comparing balanced crystalloids to saline among acutely ill adults. Two pilot studies, SPLIT (0.9\% Saline vs Plasma-Lyte 148 (PL-148) for ICU fluid Therapy) and SALT (isotonic Solution Administration Logistical Testing) demonstrated the feasibility of comparing balanced crystalloids to saline among critically ill adults, but were underpowered to detect differences in clinical outcomes. ${ }^{49,50}$ SPLIT enrolled only 84 patients with sepsis, limiting conclusions about fluid choice in this subgroup. Among the 260 patients with sepsis in SALT, the incidence of death, new renal replacement therapy, or persistent renal dysfunction was lower with the use of balanced crystalloids compared to saline ( $27.7 \%$ vs $40.8 \%$; $\mathrm{P}=0.027)$.

Two large randomized trials comparing saline to balanced crystalloids among acutely ill adults were recently completed. SMART (Isotonic Solutions and Major Adverse Renal Events Trial) compared saline to balanced crystalloids among 15,802 adult ICU patients and SALT-ED (Saline against Lactated Ringer's or Plasma-Lyte in the Emergency Department) compared saline to balanced crystalloids among 13,347 patients admitted from the emergency department to the hospital ward. Both trials demonstrated a reduction in the composite outcome of death, new renal replacement therapy, or persistent renal dysfunction with the use of balanced crystalloids compared to saline (SMART, $14.3 \%$ vs $15.4 \%$; $\mathrm{P}=$ 0.04 ; SALT-ED, $4.7 \%$ vs $5.6 \%$; $\mathrm{P}=0.01) .{ }^{51,52}$ Among the pre-specified subgroups in the SMART trial, the benefit in favor of balanced crystalloids compared to saline appeared to be greatest among patients admitted to the ICU with sepsis or septic shock. Among patients with sepsis or septic shock, the number-needed-to-treat to prevent one patient from experiencing death, new renal replacement therapy, or persistent renal dysfunction was approximately 20 patients. Given the widespread use of isotonic crystalloids for patients with sepsis, and the similar costs of saline and balanced crystalloids, these new data suggest balanced crystalloids may represent the first-line fluid therapy for adults with sepsis or septic shock (Figure 3).

\section{Colloids}

Colloid solutions contain large molecules such as starches and proteins that cannot permeate healthy capillary membranes. The theoretical benefit of such colloid solutions is improved volume expansion due to retention in the intravascular space. ${ }^{53}$ Commonly administered 
colloids include derivatives of human plasma (albumin) and semisynthetic colloids (starches, gelatins, and dextrans).

\section{Albumin}

Whether use of albumin for fluid therapy in sepsis and septic shock improves mortality remains unclear. The Saline versus Albumin Fluid Evaluation (SAFE) trial randomized nearly 7000 patients receiving fluid resuscitation to saline or $4 \%$ albumin. ${ }^{54}$ The prespecified subgroup analysis of patients with sepsis suggested that use of albumin rather than saline was safe, reduced the total volume of fluid administration, and might decrease mortality. ${ }^{55}$ The Albumin Italian Outcome Sepsis (ALBIOS) trial randomized 1,818 patients with sepsis to crystalloid alone or crystalloid and $20 \%$ albumin to maintain a serum albumin level of $30 \mathrm{~g} / \mathrm{L} .{ }^{56}$ The group assigned to receive albumin had a shorter time on vasopressors, and a post-hoc analysis of patients with septic shock at enrollment reported a lower mortality in the albumin group (RR $0.87 ; 95 \%$ CI 0.77 to 0.99 ).

A meta-analysis of five studies examining albumin therapy in sepsis showed a trend toward reduced morality with the use of albumin for sepsis resuscitation. Among patients with septic shock, the mortality benefit for albumin over crystalloids reached statistical significance.$^{57}$ In summary, the currently available evidence suggests a potential role for administration of albumin to patients with sepsis and septic shock (Figure 4), but the high relative cost of albumin has limited uptake into clinical care in the absence of definitive evidence of improved clinical outcomes. The Surviving Sepsis Campaign recommends the administration of albumin to patients with sepsis and septic shock who require "substantial amounts of crystalloids." 19 The questions of how much albumin to administer, whether to target a specific serum albumin level, and what concentration to use $(4 \%, 5 \%, 20 \%, 25 \%)$ remain unanswered.

\section{Semisynthetic colloids}

Human albumin has historically been expensive and in limited supply, which led to the development of semisynthetic colloid solutions. Gelatins are prepared by hydrolysis of bovine collagen, dextrans are biosythesized from sucrose by bacteria, and hydroxyethyl starch (HES) is synthesized from the maize-derived d-glucose polymer amylopectin. The individual colloids have different rates of loss from the circulation (determined by molecular weight) and metabolism (determined by chemical properties), which influence the duration of volume expansion. These individual properties also result in unique adverse event profiles: increased risk of acute kidney injury (HES, gelatin), allergic reactions (gelatins, dextrans), and bleeding (dextrans, HES).

Multiple trials have examined the administration of HES to patients with sepsis and do not support its use. The VISEP, ${ }^{58}$ CRYSTMAS $,{ }^{59} 6 \mathrm{~S},{ }^{60}$ and $\mathrm{CHEST}^{61}$ trials all suggested harm with the use of HES in the form of increased AKI, need for renal replacement therapy, or mortality. A subsequent meta-analysis confirmed an association between HES and both AKI and mortality. ${ }^{62}$ Recent guidelines recommend against the use of starches (HES), and suggest avoiding gelatins and dextrans as well. ${ }^{63}$ Some studies suggest that gelatins increase the risk of anaphylaxis, mortality, renal failure, and bleeding. ${ }^{64}$ Unless future research 
demonstrates a conclusive outcome benefit to a specific semisynthetic colloid among patients with sepsis, the use of all semisynthetic colloids should be avoided in sepsis management due to their risks and cost.

\section{Blood Transfusion}

For patients with anemia and sepsis or septic shock, a physiologic rationale exists for administering packed red blood cells to expand intravascular volume and provide oxygen carrying capacity. The original EGDT study by Rivers et al ${ }^{11}$ included packed red cell transfusion for patients with central venous saturation $<70 \%$ and hematocrit $<30 \%$ in the intervention group. In this trial, the intervention group experienced a lower mortality, which some authors have attributed in part to the goal-directed transfusion. ${ }^{11,12}$ In contrast, the multicenter Transfusion Requirements in Septic Shock (TRISS) trial comparing lower (hemoglobin $\leq 7 \mathrm{~g} / \mathrm{dL}$ ) versus higher (hemoglobin $\unlhd 9 \mathrm{~g} / \mathrm{dL}$ ) transfusion thresholds in patients with septic shock found no differences in clinical outcomes between groups. ${ }^{65}$ A more recent randomized single center trial compared a lower (hemoglobin $<7 \mathrm{~g} / \mathrm{dL}$ ) versus a higher (hemoglobin $<9 \mathrm{~g} / \mathrm{dL}$ ) transfusion threshold in patients with septic shock and a preexisting diagnosis of solid cancer. ${ }^{66}$ This trial reported a lower 90-day mortality with the liberal threshold. Although further evidence is required for specific subgroups of patients (e.g., those with solid cancer, severe coronary artery disease, concomitant gastrointestinal bleeding), a hemoglobin $\leq 7 \mathrm{~g} / \mathrm{dL}$ represents a reasonable threshold for red blood cell transfusion for most patients with sepsis or septic shock.

\section{SUMMARY}

Early intravenous fluid administration to patients with sepsis or septic shock corrects overt hypovolemia and may improve cardiac output, oxygen delivery, and organ function. The administration of an initial intravenous crystalloid bolus of $20 \mathrm{~mL} / \mathrm{kg}$ of body weight is a reasonable first step in the hemodynamic management of an adult patient with sepsis or septic shock. Current evidence does not suggest that early, protocolized fluid, vasopressor, and blood administration targeting hemodynamic goals improves clinical outcomes for sepsis patients in high resource settings. Moreover, fluid bolus administration to sepsis patients in low resource settings may increase the incidence of respiratory failure and death, and great caution with fluid administration should be exercised in such settings. The risks and benefits of further fluid administration after initial resuscitation should be carefully considered, potentially incorporating dynamic measures of "fluid responsiveness." Choice of intravenous fluid effects patient outcomes. Balanced crystalloids appear to lower rates of death and severe kidney dysfunction compared to saline. Albumin may improve outcomes for patients with septic shock. The side effects of semisynthetic colloids outweigh any potential benefits and they should not be administered to patients with sepsis. Additional high quality randomized trials are required to understand the optimal rate of fluid administration, optimal crystalloid composition, and how best to guide fluid therapy after initial resuscitation. Understanding the optimal approach to fluid therapy for sepsis and septic shock remains a defining challenge for critical care, and may hold the key to saving thousands of lives around the world every year. 


\section{Acknowledgments}

Source of Funding and Conflicts of Interest: M.W.S. was supported by a National Heart, Lung, and Blood Institute (NHLBI) K12 award (K12HL133117). The authors declare no potential conflicts of interest.

\section{REFERENCES}

1. Angus DC, van der Poll T. Severe Sepsis and Septic Shock. N Engl J Med 2013;369(9):840-51. [PubMed: 23984731]

2. Rhee C, Dantes R, Epstein L, et al. Incidence and Trends of Sepsis in US Hospitals Using Clinical vs Claims Data, 2009-2014. JAMA 2017;318(13):1241. [PubMed: 28903154]

3. The PRISM Investigators. Early, Goal-Directed Therapy for Septic Shock — A Patient-Level MetaAnalysis. N Engl J Med 2017;376(23):2223-34. [PubMed: 28320242]

4. Ueyama H, Kiyonaka S. Predicting the Need for Fluid Therapy_Does Fluid Responsiveness Work? J Intensive Care 2017;5:34. [PubMed: 28603624]

5. Monge García MI, Guijo González P, Gracia Romero M, et al. Effects of fluid administration on arterial load in septic shock patients. Intensive Care Med 2015;41(7):1247-55. [PubMed: 26077088]

6. Marik P, Bellomo R. A rational approach to fluid therapy in sepsis. Br J Anaesth 2016;116(3):33949. [PubMed: 26507493]

7. Cavallaro F, Sandroni C, Marano C, et al. Diagnostic accuracy of passive leg raising for prediction of fluid responsiveness in adults: systematic review and meta-analysis of clinical studies. Intensive Care Med 2010;36(9):1475-83. [PubMed: 20502865]

8. Marik PE, Cavallazzi R. Does the Central Venous Pressure Predict Fluid Responsiveness? An Updated Meta-Analysis and a Plea for Some Common Sense*: Crit Care Med 2013;41(7):1774-81. [PubMed: 23774337]

9. Leisman DE, Doerfler ME, Schneider SM, Masick KD, D'Amore JA, D'Angelo JK. Predictors, Prevalence, and Outcomes of Early Crystalloid Responsiveness Among Initially Hypotensive Patients With Sepsis and Septic Shock: Crit Care Med 2017;1.

10. Woodcock TE, Woodcock TM. Revised Starling equation and the glycocalyx model of transvascular fluid exchange: an improved paradigm for prescribing intravenous fluid therapy. $\mathrm{Br} \mathrm{J}$ Anaesth 2012;108(3):384-94. [PubMed: 22290457]

11. Rivers E, Nguyen B, Havstad S, et al. Early goal-directed therapy in the treatment of severe sepsis and septic shock. N Engl J Med 2001;345(19):1368-77. [PubMed: 11794169]

12. The Surviving Sepsis Campaign Guidelines Committee including The Pediatric Subgroup*, Dellinger RP, Levy MM, et al. Surviving Sepsis Campaign: International Guidelines for Management of Severe Sepsis and Septic Shock, 2012. Intensive Care Med 2013;39(2):165-228. [PubMed: 23361625]

13. Mouncey PR, Osborn TM, Power GS, et al. Trial of Early, Goal-Directed Resuscitation for Septic Shock. N Engl J Med 2015;372(14):1301-11. [PubMed: 25776532]

14. The ARISE Investigators and the ANZICS Clinical Trials Group. Goal-Directed Resuscitation for Patients with Early Septic Shock. N Engl J Med 2014;371(16):1496-506. [PubMed: 25272316]

15. The ProCESS Investigators. A Randomized Trial of Protocol-Based Care for Early Septic Shock. N Engl J Med 2014;370(18):1683-93. [PubMed: 24635773]

16. Maitland K, Kiguli S, Opoka RO, et al. Mortality after fluid bolus in African children with severe infection. N Engl J Med 2011;364(26):2483-2495. [PubMed: 21615299]

17. Andrews B, Muchemwa L, Kelly P, Lakhi S, Heimburger DC, Bernard GR. Simplified Severe Sepsis Protocol: A Randomized Controlled Trial of Modified Early Goal-Directed Therapy in Zambia*. Crit Care Med 2014;42(11):2315-24. [PubMed: 25072757]

18. Andrews B, Semler MW, Muchemwa L, et al. Effect of an Early Resuscitation Protocol on Inhospital Mortality Among Adults With Sepsis and Hypotension: A Randomized Clinical Trial. JAMA 2017;318(13):1233. [PubMed: 28973227] 
19. Rhodes A, Evans LE, Alhazzani W, et al. Surviving Sepsis Campaign: International Guidelines for Management of Sepsis and Septic Shock. Crit Care Med 2017;45(3):486-552. [PubMed: 28098591]

20. Boyd JH, Forbes J, Nakada T, Walley KR, Russell JA. Fluid resuscitation in septic shock: A positive fluid balance and elevated central venous pressure are associated with increased mortality*: Crit Care Med 2011;39(2):259-65. [PubMed: 20975548]

21. Neyra JA, Li X, Canepa-Escaro F, et al. Cumulative Fluid Balance and Mortality in Septic Patients With or Without Acute Kidney Injury and Chronic Kidney Disease. Crit Care Med 2016;44(10): 1891-900. [PubMed: 27352125]

22. Semler MW, Wheeler AP, Thompson BT, Bernard GR, Wiedemann HP, Rice TW. Impact of Initial Central Venous Pressure on Outcomes of Conservative Versus Liberal Fluid Management in Acute Respiratory Distress Syndrome: Crit Care Med 2016;1.

23. The CLASSIC Trial Group, The Scandinavian Critical Care Trials Group, Hjortrup PB, et al. Restricting volumes of resuscitation fluid in adults with septic shock after initial management: the CLASSIC randomised, parallel-group, multicentre feasibility trial. Intensive Care Med 2016;42(11):1695-705. [PubMed: 27686349]

24. Hjortrup PB, Haase N, Wetterslev J, et al. Effects of fluid restriction on measures of circulatory efficacy in adults with septic shock. Acta Anaesthesiol Scand 2017;61(4):390-8. [PubMed: 28150304]

25. Velissaris D, Pierrakos C, Scolletta S, De Backer D, Vincent JL. High mixed venous oxygen saturation levels do not exclude fluid responsiveness in critically ill septic patients. Crit Care 2011;15(4):R177. [PubMed: 21791090]

26. Marik PE, Cavallazzi R, Vasu T, Hirani A. Dynamic changes in arterial waveform derived variables and fluid responsiveness in mechanically ventilated patients: A systematic review of the literature*: Crit Care Med 2009;37(9):2642-7. [PubMed: 19602972]

27. Marik PE, Monnet X, Teboul J-L. Hemodynamic parameters to guide fluid therapy. Ann Intensive Care 2011;1(1):1. [PubMed: 21906322]

28. Yang X, Du B. Does pulse pressure variation predict fluid responsiveness in critically ill patients? A systematic review and meta-analysis. Crit Care Lond Engl 2014;18(6):650.

29. Lu N, Xi X, Jiang L, Yang D, Yin K. Exploring the best predictors of fluid responsiveness in patients with septic shock. Am J Emerg Med 2017;35(9):1258-61. [PubMed: 28363617]

30. Long E, Oakley E, Duke T, Babl FE. Does Respiratory Variation in Inferior Vena Cava Diameter Predict Fluid Responsiveness: A Systematic Review and Meta-Analysis. SHOCK 2017;47(5):550_ 9. [PubMed: 28410544]

31. Alhashemi JA, Cecconi M, Hofer CK. Cardiac output monitoring: an integrative perspective. Crit Care Lond Engl 2011;15(2):214.

32. Zhang Z, Ni H, Qian Z. Effectiveness of treatment based on PiCCO parameters in critically ill patients with septic shock and/or acute respiratory distress syndrome: a randomized controlled trial. Intensive Care Med 2015;41(3):444-51. [PubMed: 25605469]

33. Kuan WS, Ibrahim I, Leong BSH, et al. Emergency Department Management of Sepsis Patients: A Randomized, Goal-Oriented, Noninvasive Sepsis Trial. Ann Emerg Med 2016;67(3):367-378.e3. [PubMed: 26475246]

34. Hammond NE, Taylor C, Finfer S, et al. Patterns of intravenous fluid resuscitation use in adult intensive care patients between 2007 and 2014: An international cross-sectional study. PloS One 2017;12(5):e0176292. [PubMed: 28498856]

35. McIntyre L, Rowe BH, Walsh TS, et al. Multicountry survey of emergency and critical care medicine physicians' fluid resuscitation practices for adult patients with early septic shock. BMJ Open 2016;6(7):e010041.

36. Awad S, Allison SP, Lobo DN. The history of 0.9\% saline. Clin Nutr Edinb Scotl 2008;27(2):17988 .

37. Asfar P, Schortgen F, Boisramé-Helms J, et al. Hyperoxia and hypertonic saline in patients with septic shock (HYPERS2S): a two-by-two factorial, multicentre, randomised, clinical trial. Lancet Respir Med 2017;5(3):180-190. [PubMed: 28219612] 
38. Waters JH, Gottlieb A, Schoenwald P, Popovich MJ, Sprung J, Nelson DR. Normal saline versus lactated Ringer's solution for intraoperative fluid management in patients undergoing abdominal aortic aneurysm repair: an outcome study. Anesth Analg 2001;93(4):817-822. [PubMed: 11574339]

39. Kellum JA. Fluid resuscitation and hyperchloremic acidosis in experimental sepsis: improved short-term survival and acid-base balance with Hextend compared with saline. Crit Care Med 2002;30(2):300-305. [PubMed: 11889298]

40. Hasman H, Cinar O, Uzun A, Cevik E, Jay L, Comert B. A randomized clinical trial comparing the effect of rapidly infused crystalloids on acid-base status in dehydrated patients in the emergency department. Int J Med Sci 2012;9(1):59-64. [PubMed: 22211091]

41. Chowdhury AH, Cox EF, Francis ST, Lobo DN. A Randomized, Controlled, Double-Blind Crossover Study on the Effects of 2-L Infusions of 0.9\% Saline and Plasma-Lyte ${ }^{\circledR} 148$ on Renal Blood Flow Velocity and Renal Cortical Tissue Perfusion in Healthy Volunteers: Ann Surg 2012;256(1):18-24. [PubMed: 22580944]

42. Potura E, Lindner G, Biesenbach P, et al. An Acetate-Buffered Balanced Crystalloid Versus 0.9\% Saline in Patients with End-Stage Renal Disease Undergoing Cadaveric Renal Transplantation: A Prospective Randomized Controlled Trial. Anesth Analg 2015;120(1):123-9. [PubMed: 25185593]

43. Orbegozo D, Su F, Santacruz C, et al. Effects of Different Crystalloid Solutions on Hemodynamics, Peripheral Perfusion, and the Microcirculation in Experimental Abdominal Sepsis. Anesthesiology 2016;125(4):744-54. [PubMed: 27655180]

44. Jaynes MP, Murphy CV, Ali N, Krautwater A, Lehman A, Doepker BA. Association between chloride content of intravenous fluids and acute kidney injury in critically ill medical patients with sepsis. J Crit Care 2018;44:363-7. [PubMed: 29288964]

45. Kellum JA, Song M, Almasri E. Hyperchloremic Acidosis Increases Circulating Inflammatory Molecules in Experimental Sepsis. Chest 2006;130(4):962-7. [PubMed: 17035425]

46. Shaw AD, Bagshaw SM, Goldstein SL, et al. Major complications, mortality, and resource utilization after open abdominal surgery: 0.9\% saline compared to Plasma-Lyte. Ann Surg 2012;255(5):821-829. [PubMed: 22470070]

47. Shaw AD, Raghunathan K, Peyerl FW, Munson SH, Paluszkiewicz SM, Schermer CR. Association between intravenous chloride load during resuscitation and in-hospital mortality among patients with SIRS. Intensive Care Med 2014;40(12):1897-905. [PubMed: 25293535]

48. Raghunathan K, Bonavia A, Nathanson BH, et al. Association between initial fluid choice and subsequent in-hospital mortality during the resuscitation of adults with septic shock. Anesthesiol $\mathrm{J}$ Am Soc Anesthesiol 2015;123(6):1385-1393.

49. Young P, Bailey M, Beasley R, et al. Effect of a Buffered Crystalloid Solution vs Saline on Acute Kidney Injury Among Patients in the Intensive Care Unit: The SPLIT Randomized Clinical Trial. JAMA 2015;314(16):1701. [PubMed: 26444692]

50. Semler MW, Wanderer JP, Ehrenfeld JM, et al. Balanced Crystalloids versus Saline in the Intensive Care Unit. The SALT Randomized Trial. Am J Respir Crit Care Med 2017;195(10):1362-72. [PubMed: 27749094]

51. Semler MW, Self WH, Wanderer JP, et al. Balanced Crystalloids versus Saline in Critically Ill Adults. N Engl J Med 2018;378(9):829-39. [PubMed: 29485925]

52. Self WH, Semler MW, Wanderer JP, et al. Balanced Crystalloids versus Saline in Noncritically Ill Adults. N Engl J Med 2018;378(9):819-28. [PubMed: 29485926]

53. Sánchez M, Jiménez-Lendínez M, Cidoncha M, et al. Comparison of fluid compartments and fluid responsiveness in septic and non-septic patients. Anaesth Intensive Care 2011;39(6):1022-9. [PubMed: 22165353]

54. Finfer S, Bellomo R, Boyce N, et al. A comparison of albumin and saline for fluid resuscitation in the intensive care unit. N Engl J Med 2004;350(22):2247-56. [PubMed: 15163774]

55. The SAFE Study Investigators. Impact of albumin compared to saline on organ function and mortality of patients with severe sepsis. Intensive Care Med 2011;37(1):86-96. [PubMed: 20924555] 
56. Caironi P, Tognoni G, Masson S, et al. Albumin Replacement in Patients with Severe Sepsis or Septic Shock. N Engl J Med 2014;370(15):1412-21. [PubMed: 24635772]

57. Xu J-Y, Chen Q-H, Xie J-F, et al. Comparison of the effects of albumin and crystalloid on mortality in adult patients with severe sepsis and septic shock: a meta-analysis of randomized clinical trials. Crit Care 2014;18:702. [PubMed: 25499187]

58. Brunkhorst FM, Engel C, Bloos F, et al. Intensive Insulin Therapy and Pentastarch Resuscitation in Severe Sepsis. N Engl J Med 2008;358(2):125-39. [PubMed: 18184958]

59. Guidet B, Martinet O, Boulain T, et al. Assessment of hemodynamic efficacy and safety of $6 \%$ hydroxyethylstarch $130 / 0.4$ vs. $0.9 \% \mathrm{NaCl}$ fluid replacement in patients with severe sepsis: The CRYSTMAS study. Crit Care 2012;16:R94. [PubMed: 22624531]

60. Perner A, Haase N, Guttormsen AB, et al. Hydroxyethyl Starch 130/0.42 versus Ringer's Acetate in Severe Sepsis. N Engl J Med 2012;367(2):124-34. [PubMed: 22738085]

61. Myburgh JA, Finfer S, Bellomo R, et al. Hydroxyethyl Starch or Saline for Fluid Resuscitation in Intensive Care. N Engl J Med 2012;367(20):1901-11. [PubMed: 23075127]

62. Zarychanski R, Abou-Setta AM, Turgeon AF, et al. Association of Hydroxyethyl Starch Administration With Mortality and Acute Kidney Injury in Critically Ill Patients Requiring Volume Resuscitation: A Systematic Review and Meta-analysis. JAMA 2013;309(7):678-88. [PubMed: 23423413]

63. Joannidis M, Druml W, Forni LG, et al. Prevention of acute kidney injury and protection of renal function in the intensive care unit: update 2017: Expert opinion of the Working Group on Prevention, AKI section, European Society of Intensive Care Medicine. Intensive Care Med 2017;43(6):730-49. [PubMed: 28577069]

64. Moeller C, Fleischmann C, Thomas-Rueddel D, et al. How safe is gelatin? A systematic review and meta-analysis of gelatin-containing plasma expanders vs crystalloids and albumin. J Crit Care 2016;35:75-83. [PubMed: 27481739]

65. Holst LB, Haase N, Wetterslev J, et al. Lower versus Higher Hemoglobin Threshold for Transfusion in Septic Shock. N Engl J Med 2014;371(15):1381-91. [PubMed: 25270275]

66. Bergamin FS, Almeida JP, Landoni G, et al. Liberal Versus Restrictive Transfusion Strategy in Critically Ill Oncologic Patients: The Transfusion Requirements in Critically Ill Oncologic Patients Randomized Controlled Trial*. Crit Care Med 2017;45(5):766-73. [PubMed: 28240687] 


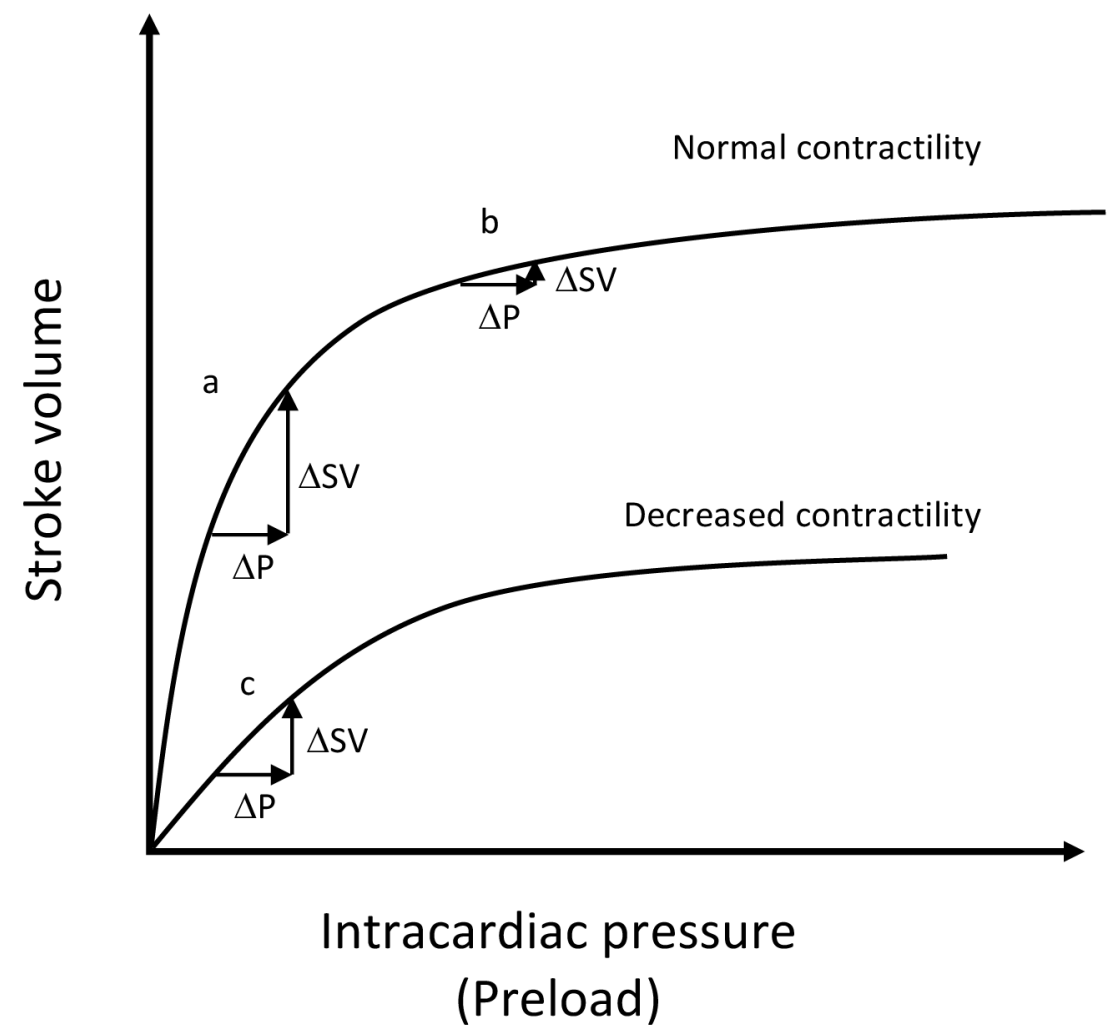

Figure 1. Relationship between intracardiac pressure and stroke volume according to the Frank Starling Curve

The same increase in preload $(\Delta \mathrm{P})$ can either cause a significant increase in stroke volume $(\Delta \mathrm{SV})(\mathbf{a})$, or no significant increase in stroke volume $(\mathbf{b}, \mathbf{c})$ depending on the initial preload (starting point on the curve) (b) or the shape of the curve, such as a flattened curve from decreased contractility (c). Unfortunately, this simple view fails to capture all the ways in which intravenous fluid may affect stroke volume. P, preload; SV, stroke volume. 


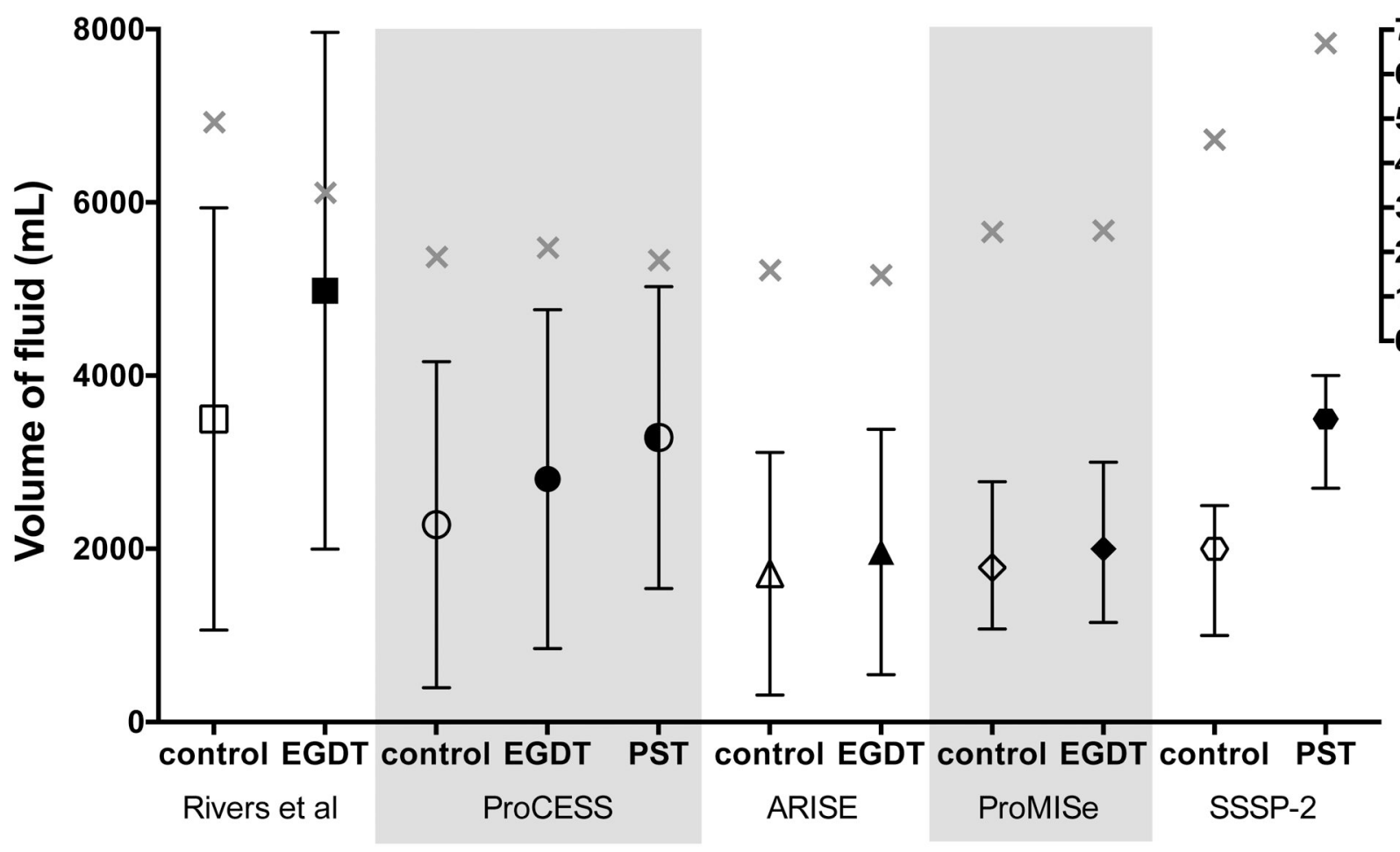

Figure 2. Fluid administration in EGDT trials

Volume of intravenous fluid during the first 6 hours in each EGDT trial, including preenrollment fluid. Volume is presented as mean and standard deviation for all trials except ProMISe and SSSP-2, which are median and interquartile range. Mortality (gray X) is through 60 days in ProCESS and 28 days in all other trials. PST, protocol-based standard therapy or sepsis protocol therapy. 


\section{Balanced Crystalloids vs Saline in ICU Patients with Sepsis}

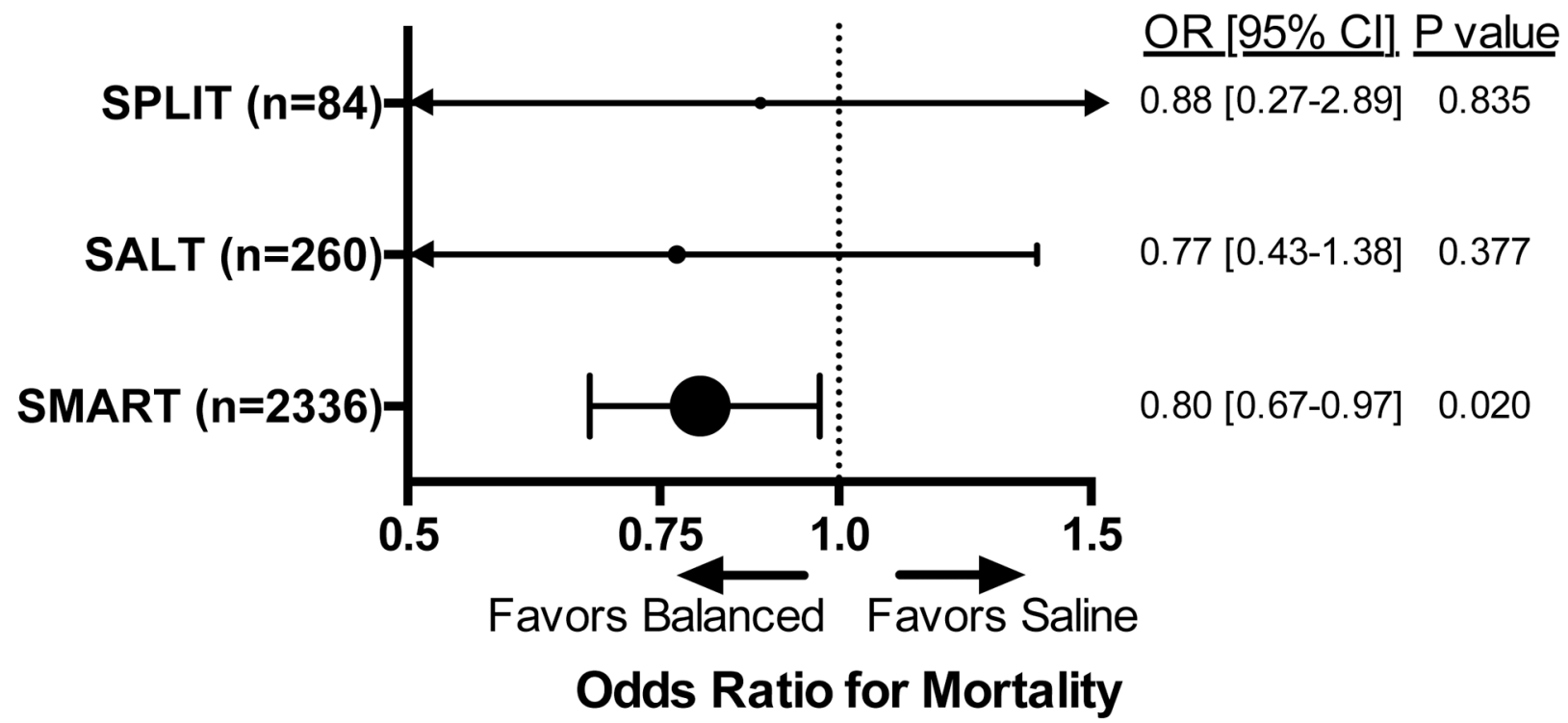

Figure 3. Odds of death with use of balanced crystalloids vs saline for ICU patients with sepsis SPLIT, ${ }^{49}$ SALT $^{50}$ and SMART ${ }^{51}$ are three randomized trials comparing the use of balanced crystalloids versus saline in the ICU. Odds ratios of in-hospital mortality are shown for SPLIT, odds ratios of 30-day in-hospital mortality are shown for SALT and SMART. 


\section{Albumin vs Crystalloid in Septic Shock}

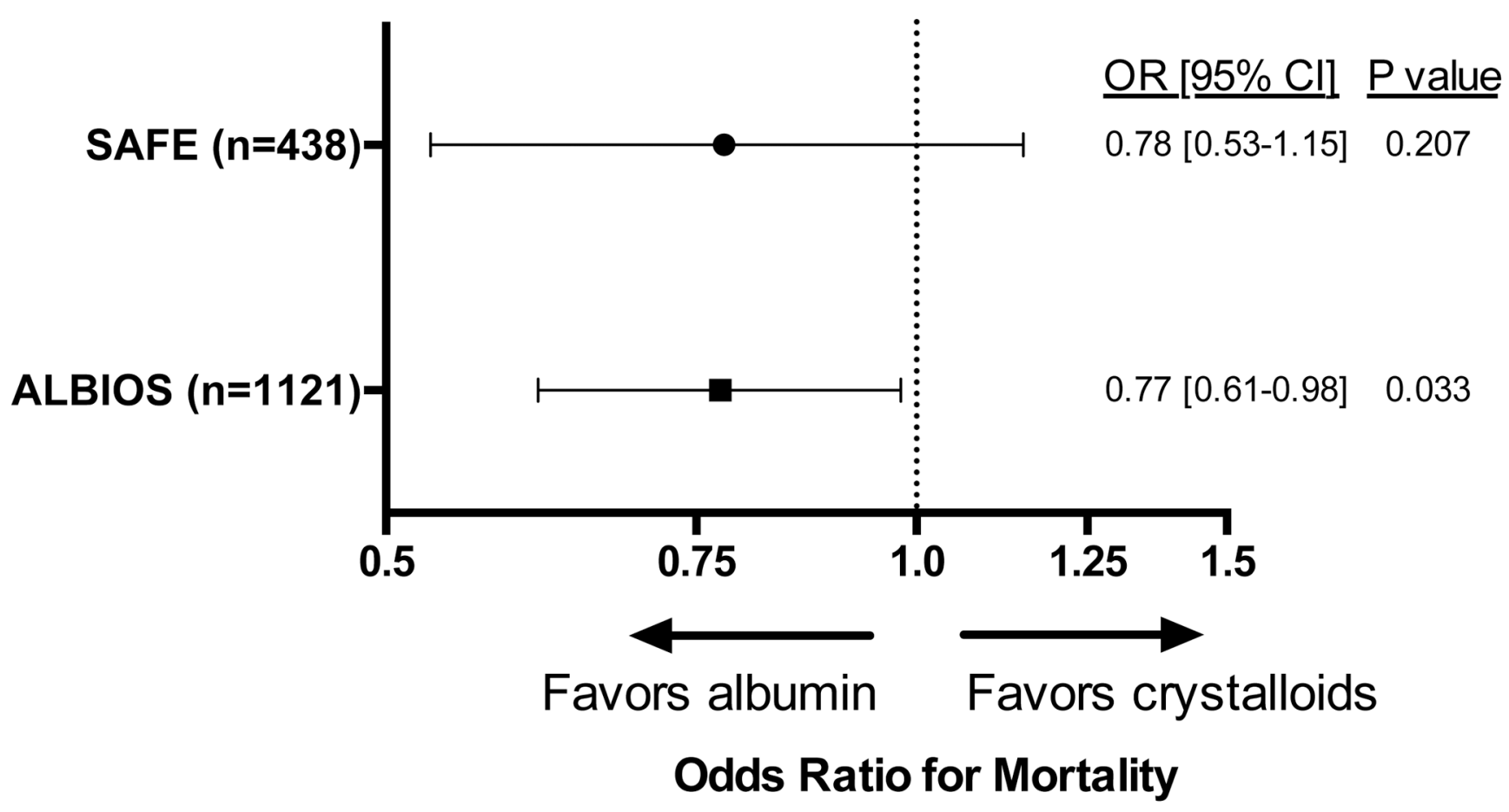

Figure 4. Odds of death with albumin use in septic shock

Odds ratios for 90-day mortality are displayed for albumin compared to crystalloid for patients with septic shock. Subgroup analysis of patients with septic shock from the SAFE ${ }^{55}$ and ALBIOS $^{56}$ trials suggests a possible mortality benefit with albumin use. In the SAFE study, patients in the albumin group were given $4 \%$ albumin rather than crystalloid. In the ALBIOS study, patients in the albumin group were given $20 \%$ albumin in addition to crystalloid to maintain a serum level of $30 \mathrm{~g} / \mathrm{L}$. 


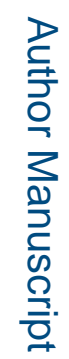
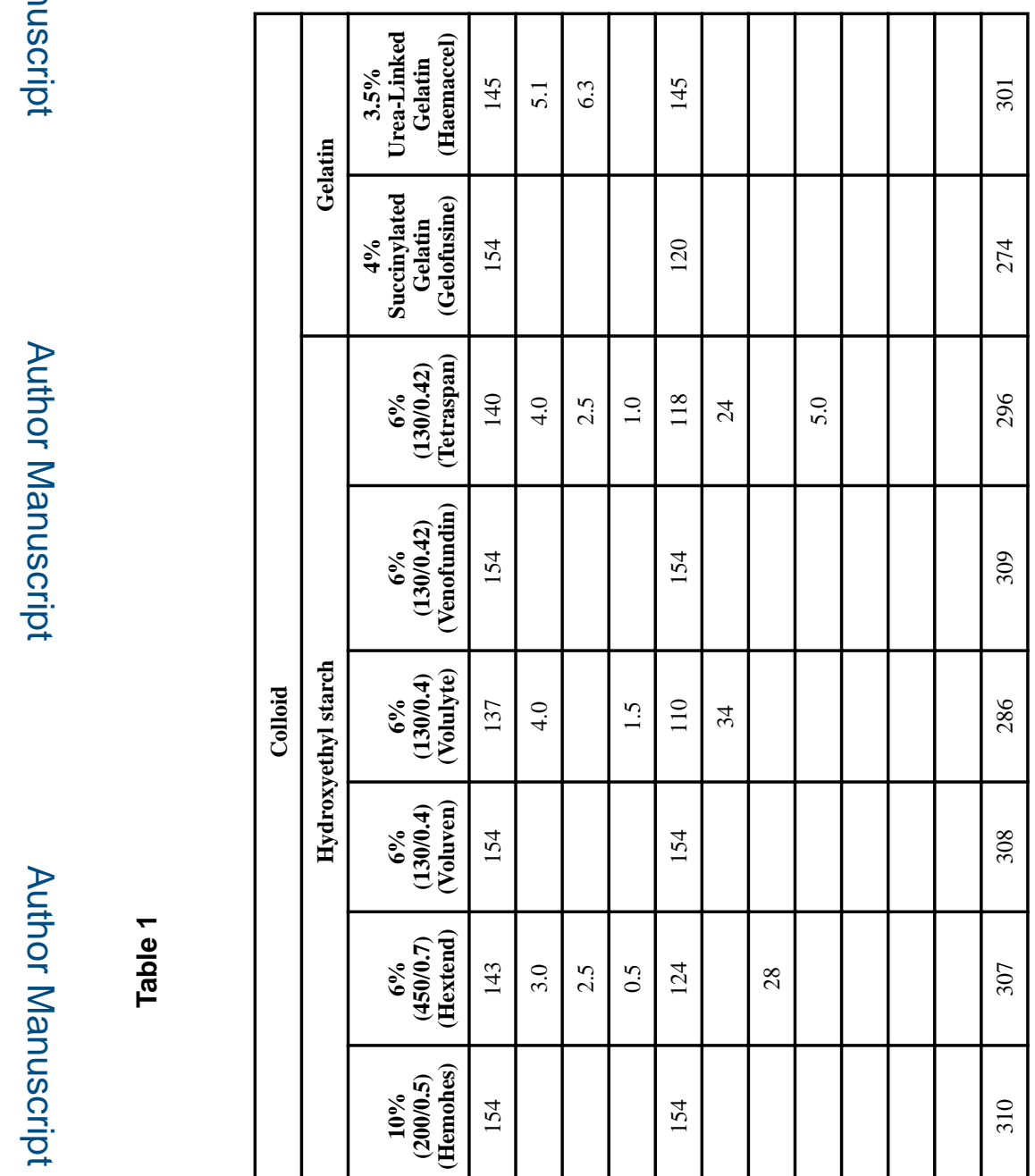

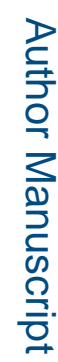

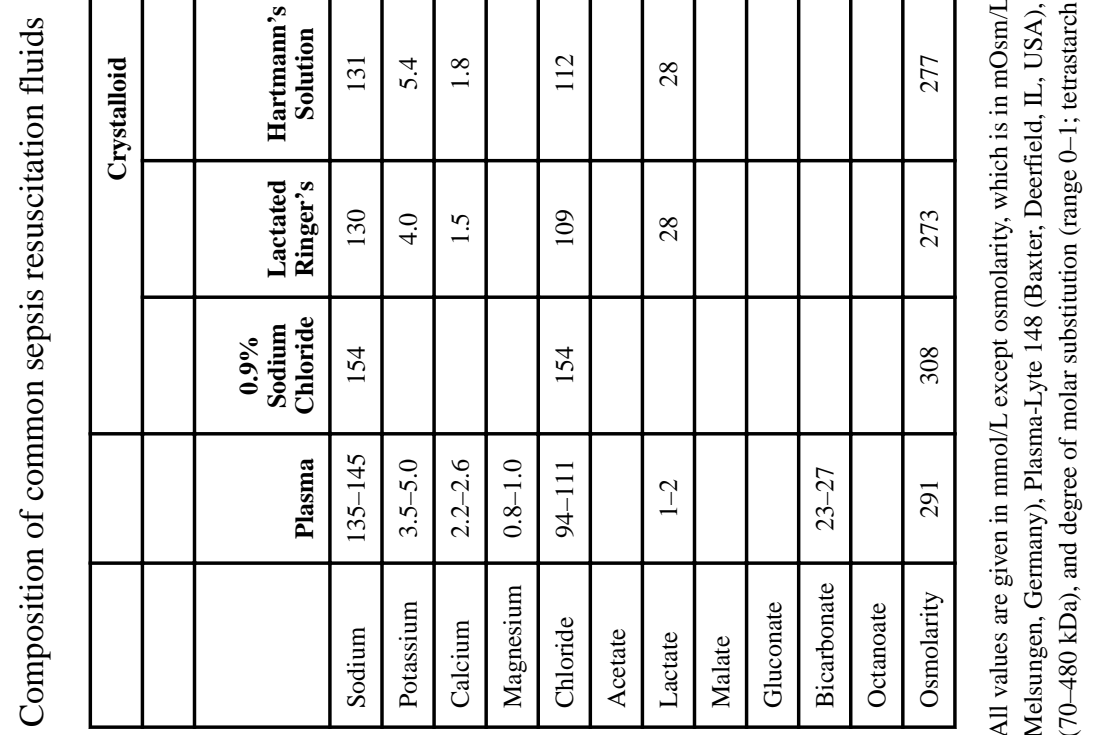

